\section{Unterschiedliche Behandlung am Lebensende}

\begin{abstract}
In einer Kohortenstudie fanden sich geschlechtsspezifische Unterschiede bei der Behandlung von Krebspatienten in ihrer letzten Lebensphase. Eine empathische, aber realistische Beratung kann die Entscheidung gegen eine aggressive „end of life"-Behandlung beeinflussen.
\end{abstract}

V iele Krebspatienten erhalten am Ende des Lebens (End of Life, EoL) trotz des absehbaren Todes eine aggressive Behandlung mit Intensivversorgung und neuer Chemotherapie. Selten wird dadurch die Lebenszeit verlängert oder die Lebensqualität verbessert.

Ein zentraler Punkt für die Entscheidung zugunsten einer solchen Behandlung ist die Kommunikation zwischen Ärzten, Patienten und Angehörigen. Kleinere Studien zeigen, dass es geschlechtsspezifische Unterschiede in der Einstellung zu Sterben und Tod sowie dem Verhalten in dieser Lebensphase gibt - Frauen entscheiden sich eher gegen eine aggressive Behandlung als Männer. In eine prospektive Kohortenstudie wurden 353 Patienten mit metastasiertem Krebs im Endstadium aufgenommen, die im Median 4,1 Monate vor dem Tod an einer Befragung zu möglichen Prädiktoren für die EoL-Behandlung teilgenommen hatten und bei denen nach ihrem Tod Daten zur EoL-Betreuung vorlagen.

Frauen lehnten demnach häufiger Maßnahmen zur Wiederbelebung ab (48 vs. $37 \%$; $=0,039)$ und bevorzugten eine palliative EoL-Betreuung (70 vs. $58 \%$; $p=0,017)$. Umgekehrt waren Patienten, die am Lebensende intensivmedizinisch behandelt wurden, eher männlich (73 vs. $52 \%$; $=0,015)$ und lehnten eine Palliativbehandlung ab (35 vs. $67 \%$; $p<0,001$ ). Nach Berücksichtigung von Störfaktoren verbrachten Männer, die vorher zur Behandlung am Lebensende beraten worden waren, seltener die letzte Lebenswoche auf der Intensivstation als Männer, die nicht beraten worden waren (adjustierte Odds Ratio 0,26; $p=0,04)$. Bei Frauen hatte die Beratung keinen Einfluss.

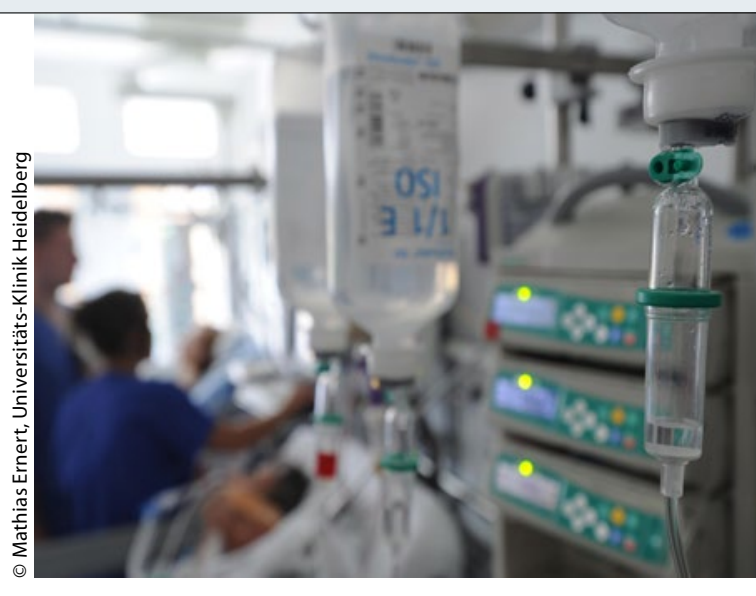

Männer werden am Lebensende öfter intensivmedizinisch betreut.

Fazit: Männer im finalen Krebsstadium erhalten häufiger als Frauen eine intensivmedizinische Behandlung, die die Lebensqualität vermindern kann und die Lebensspanne normalerweise kaum nennenswert verlängert. Eine eingehende Beratung reduziert den Wunsch nach aggressiver Therapie. Bei Frauen besteht auch ohne Beratung die Tendenz zu einer eher palliativen EoL-Behandlung.

Barbara Kreutzkamp

Sharma RK et al. Male-female patient differences in the association between end-of-life discussions and receipt of intensive care near death. Cancer. 2015;121(16):2814-20.

\title{
Wann das Kribbeln chronisch wird
}

\begin{abstract}
Neuropathien nach Oxaliplatin-Therapie sind häufig. Dennoch war über genaue Symptome, Abklingen und Chronifizierung der Par- und Dysästhesien bisher wenig bekannt. In einer US-Studie wurden die Details analysiert.
\end{abstract}

$\mathrm{N}$ europathien sind häufig Folge einer Oxaliplatin-Therapie. Akute Parästhesien oder Dysästhesien sind meist binnen Tagen reversibel, doch kann auch eine spätere, langanhaltende Neurotoxizität mit einer Überempfindlichkeit entstehen, die die Lebensqualität einschränkt. Viele Fragen sind in diesem Zusammenhang ungeklärt: Welche Faktoren begünstigen welche Beschwerden? Ist jede Parästhesie oder Dysästhesie reversibel? Welche Symptome treten genau auf? Und sind Schwere und Form der initialen Neuropathien prognostisch für weitere Neuropathien? In einer US-Studie wurde das Auftreten von Neuropathien bei Patienten evaluiert, die adjuvant FOLFOX (Fluorouracil, Folinsäure,
Oxaliplatin) erhielten. In jedem Zyklus wurden die akuten Symptome erfasst und über 18 Monate zudem bei Patienten mit peripherer Neuropathie vor und nach jedem Zyklus die chronische Neurotoxizität mithilfe des EORTC-Lebensqualitätsfragebogens ermittelt.

308 der 346 Patienten (89\%) zeigten im ersten Zyklus mindestens ein Symptom einer akuten Neuropathie (72\% Überempfindlichkeit beim Berühren kalter Gegenstände, 71 \% Empfindlichkeit beim Schlucken kalter Getränke oder Speisen, $62 \%$ Unwohlsein in der Kehle, $42 \%$ Muskelkrämpfe). Akute Symptome waren an Tag 3 am ausgeprägtesten und besserten sich dann, gingen aber zwischen den Behandlungen nicht immer vollständig zu- rück. Traten dieselben Symptome auch in den Zyklen 2-12 auf, waren sie etwa doppelt so schwer wie in Zyklus 1. Von den chronischen Symptomen war Kribbeln das schwerwiegendste, gefolgt von Taubheit und Schmerzen. Während der Chemotherapie waren Symptome in den Händen prominenter als in den Füßen; nach 18 Monaten waren die Füße jedoch stärker betroffen als die Hände. Eine schwerere akute Neuropathie während des ersten Zyklus chronifizierte später häufiger ( $\mathrm{p}<0,0001)$.

Fazit: Akute, durch Oxaliplatin induzierte neuropathische Symptome bilden sich zwischen den Behandlungszyklen nicht immer vollständig zurück. Die Schwere der akuten Neuropathie korreliert mit einer chronischen Neurotoxizität.

Christina Berndt

Pachman DR et al. Clinical Course of OxaliplatinInduced Neuropathy: Results From the Randomized Phase III Trial N08CB (Alliance). J Clin Oncol. 2015;33(30):3416-22. 